\title{
FACILITATING HOSPITAL ACTIVITIES WITH INFORMATION TECHNOLOGY CASE STUDY: BLOOD COLLECTION AND TRANSFUSION IN FARYAB CENTRAL HOSPITAL
}

\author{
Ghulam Rasoul Zaini \\ Department of Computer Science \\ Faryab University, Faryab Province, Afghanistan
}

\begin{abstract}
Like other organization in the province, Faryab provincial hospital uses the IT and Internet features in an inefficient manner for their activities. The hospital uses social media to announce the requirement of the blood when it's necessary for some treatment or for some other urgent operations. While, these announces most of the times may not be able to reach relevant bodies. Henceforth, author of the paper is attempting to design a specific platform which will use the Internet service by the hospital for their activities in an efficient way. This platform can be used to facilitate blood collection, vaccine operations and other hospital activities.
\end{abstract}

Keywords - Faryab Province, public health department, Facebook Graph API, Google Map API, hospital, social media

\section{INTRODUCTION}

Recently, Afghanistan, being one of the least developed countries, has seen progresses in the field of technology and it has applied its features including those of the Internet. Regarding [1], there exists a rapid growth of the Internet use by the people starting from 0.004 percent in 2001 up to 11.44 percent in 2017. While in most cases, social media has a significant part of usage. The [2] states that $95 \%$ of social media users sampled have a Facebook account, compared to other platforms such as Twitter with a $6 \%$ usage rate, Instagram with $10 \%$ usage rate. Instant Messaging platforms however also proven to be very popular. However, it's not used for the purposes of online shopping, to facilitate home appliances, transportation, and other related aspects of modern IT world. The reason can be traced to a plenty of transmission channels; one might be that people are not able to efficiently use these platforms. Due to a low-level technical efficiency, the use of IT is limited to its simplest forms and avoid more progressed and complex phases therewith.
In Faryab province, majority of the population employs the internet and social media in their basic forms. Forms including printing and editing photos, writing official documents, Facebook to collect relevant local news and public awareness. Some organization use the database as well, e.g., Ms. Excel, Ms. Access, as a primary form to manage part of their official data and for basic calculation purposes. The public health department in Faryab province, the same as other organization, is using the Internet and some other IT facilities to perform some daily and routine tasks in a basic form. Unfortunately, in some situation, the IT facilities and Internet are being accused.

In most of the cases, in most of the organizations. employees or computer workers including those of Faryab hospital are not well aware how to use computer and the Internet in their best manner possible. They are using one application to a specific functionality while it can be performed quickly and efficiently with another application as well. In the same way, The Internet usage is very limited and basic.

However, instead of using the Internet in a broad and general manner, there is enough space to design and develop a specific application that would solely target facilitating hospital's use of IT for the purposes of its activities. In Faryab hospital, for example, the collection of blood for transfusion purpose is not being managed in an efficient way and mostly the necessary blood for patients even in an urgent circumstance, is being announced by social media, while it is not a right approach.

Based on the studies I have gone through and considering the hospital facilities, I found that designing a specific system or computer application for this specific purpose is the right approach rather than using social media. In this paper, I have attempted to design a prototype for a specific computer application for this purpose. At the second section, it's explained that how the problem tackled through designing a 


\section{International Journal of Engineering Applied Sciences and Technology, 2019 \\ Vol. 4, Issue 6, ISSN No. 2455-2143, Pages 333-336 \\ Published Online October 2019 in IJEAST (http://www.ijeast.com)}

system, followed by an explanation of the system architecture in the third section. Finally, I concluded the paper with the explanation of future works and the system scalabilities.

\section{PROBlRm AND SUGGeSTED SOLUTION}

In the Faryab central hospital like other hospitals, more critical treatments and other operations occur, while the hospital is supporting other 14 district hospitals as well, and some treatments which are not possible in the district hospitals are being practiced in the central hospital [3]. The Basic Packages of Health Services (BPHS) places an appropriate emphasis on basic primary health care, while prioritizing the building and equipping of hospital facilities that can deliver emergency obstetric care. There are four major donors in the health sector: USAID, World Bank, EC and UNICEF (which is both a donor and implementer of projects)[4, p. 129]. In addition to deliver health care services, they also enhance facilitates like health information system and other services and operations in the hospital [5]. One of the vital operations occurring in the hospital is the blood transfusion which requires a fully equipped blood bank. But unfortunately, the blood bank in the hospital is not fully equipped to provide services as expected. Traditionally, like other hospitals, the blood transfusion operation to do some treatments or other operations can be done by earning the necessary blood from blood donors or someone volunteers, in most cases, the relevance of the patient. To collect the necessary blood, often, they are asking the blood donors, the volunteers through social media by posting with journalists' Facebook accounts or other more available Facebook pages. Although, it is working even in an inefficient way, due to being Facebook a frequently used social media, and it is accessible to most people. However, its work is in passive mode and requires to be seen by the volunteers. The blood donors must have to see the post and donate the necessary blood to the patients or for the treatments. They may do not see the post in most cases. Therefore, in some situation, the blood transfusion may not occur quickly and easily, while, it is very important and essential for patient care and treatments.

This blood collection approach is not the right way, because the nearest and blood donors may not see the Facebook post, even they can donate the necessary blood. Moreover, it can be seen by unrelated and not nearest users who cannot do anything accordingly. Therefore, this approach is not suitable for such an emergency and urgent situation. In some circumstances will cause to fail the treatments or to be unavailable the necessary blood for patients or delay of the blood transfusion. However, the blood collection to happen as quickly, blood donors should be informed as soon as possible.

This work suggests to designe and develop a specific system or application to overcome this problem. The suggested Blood Collector Messenger System (BCMS) works in an active mode by broadcasting the message to blood donors and volunteers quickly within a specific geolocational area which can be determined by some technique explained in the following section. This system detects the nearest and related people by their mobile devices' GPS service, and broadcast the message for them, and they can then make respond to the message as quickly to donate the necessary blood. The following section explains the architecture of the system.

\section{SYSTEM ARCHITECTURE}

The BCMS system is designed in a Peer-to-Peer approach where one peer is the hospital side that sends message to other side/peer where the users' mobile located in a specific geolocational area receives the message. The content of the message is blood type, its amount, time to be delivered or additional note the hospital side or the sender generates. When a user receives the message, he/she can response through his/her mobile message application (in this case Facebook messenger) or he/she can ignore it. So, a live conversation between sender (system user or hospital side) and receivers (blood donors) is established, and it can help the blood collection procedure to be done timely and quickly. When users respond, his/her message along his/her mobile location and user account information will be delivered to the sender hospital side as illustrated in the Fig. 1 .

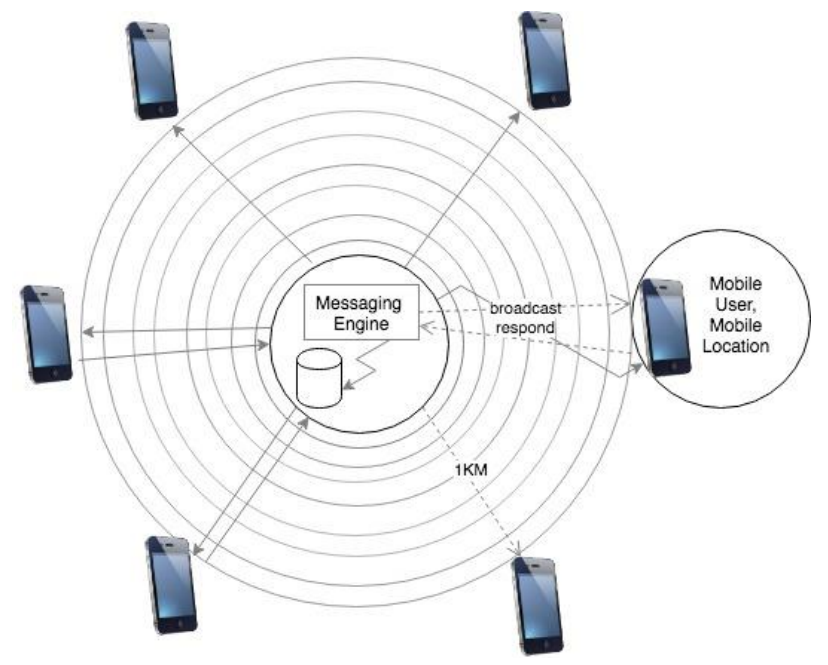

Fig. 1. BCMS Architecture

BCMS system can be developed in different platforms, web application that will be used by hospital user sat down behind one computer or as mobile application that will be used by a responsible person. It uses the Facebook Graph API [6], Google Map APIs [7], Facebook Message Platform [8] and PubNub technologies. Google Map API determines users' devices location especially the mobile devices within the range of area specified by the system users, gathers useful information from the devices and feeds the information to other parts of the system. Then the system uses this collected 


\section{International Journal of Engineering Applied Sciences and Technology, 2019 \\ Vol. 4, Issue 6, ISSN No. 2455-2143, Pages 333-336 \\ Published Online October 2019 in IJEAST (http://www.ijeast.com)}

information to indicate the nearest people devices in a specific range defined by the system's users and messages them [9]. In this architecture, the distance is defined as $1 \mathrm{KM}$ by default, which is realistic to inform the nearest people for the necessary blood and collection operation. This distance is also better for the system performance, the less distance requires less processing, but users can define the distance as they want regarding the situation. The PubNub technologies will be used to track the devices' real-time location with the help of the geolocation feature of the Google Map APIs [10] [11]. PubNub is a programmable network for developing real-time applications and an evolution from three-tier architecture, purpose-built to handle all the complexities of data streams. PubNub operates at the edge of the network to handle and apply logic to real-time data, thereby minimizing latency to 250 milliseconds or less worldwide, and guaranteeing reliability and scalability [12]. Through these integrated components and using the Facebook Graph API, it is possible to use the Facebook IDs from the detected devices to message while the Facebook messaging is popular among people. As [2] states that 95 percent of social media users in Afghanistan have a Facebook account. The Facebook Message Platform is used to message to the dedicated devices and communicate the sender and receiver with each other through the message even if they are not friends in Facebook [13] [8].

BCMS system, in addition, to receive the volunteers' messages when they replied, it can store their information in the system or any storage device. These collected and stored information is very important to the hospital and can then be used for some purposes later, like as statistical information of blood type of the people, monthly earned blood, how much blood can a person typically give to a patient and etc. The amount of the data will be timely increased and becomes massive. With this massive data and information collected by the system, the hospital can estimate its blood transfusion operation monthly or weekly bases and determine blood demand for its operation and plan its activities more efficiently and can make the right decision when needed.

In addition, this massive gathered information can also be used to filter message receivers of the system, and the system based on this data then can decide to do messages to which nearest people those have actually necessary blood for the patient. And this filtering functionality of the system will be better by time passing, but in the beginning, the system will broadcast the message to everyone who is within a specified area though. At the beginning, the sent messages may have some boring to somebodies, but it can be avoided sending messages later to unrelated persons based on information which exist within the system. Thus, the system operation can also be enhanced later, and the system will have better performance by filtering and reducing the number of receivers that have unrelated blood type to the patient.

\section{CONCLUSION}

Using the technology features and Internet in an efficient way, hospitals and other organizational units facilitates their activities and other daily and routinely works. Like this system, there is always possible to use technologies and Internet for other hospital activities to facilitate and enhance its functionalities.

The system is not only be used to message such a situation in the hospital but can be used in another situation as well. It can be used to announce vaccine operations in a specific geolocational area of the beneficiaries for example and the vaccine operators do the vaccine operations quickly as possible. It has to be mentioned that the announcement of vaccine operation is going on by the announcing in the mosques' in most area, likely in rural area, but it is not suitable nowadays where the most rural area is becoming small cities where the traffic noises are increasing day-to-day and other mechanic systems are interrupting the mosques' load system which was useful and functional in the past. The messaging approach is more functional in such a situation rather than the traditional.

Although, developing the system with the web-based application is useful and functional in such organizations where the staffs and computer operators usually are using desktop computers, but the system can also be developed in varies version in addition to the web-based application, like android version, iOS version or any other platforms, while the mobile users are rapidly growing up from 5.8 percent to 21.2 percent since 2007 [1].

\section{REFERENCE}

[1] Central Statistics Organization, "Afghanistan Provincial Profile." National Statistics and Information Authority, 2018.

[2] Altai Consulting for Internews, "Social Media in Afghanistan -Users and Engagement." USAID, 2017.

[3] Ghulam Farooq et al., "A Guide to Government in Afghanistan Case Study: Faryab Province," AREU \& The World Bank, 2004.

[4] A. Wilder, A. Tully, N. Manning, Y. Osmani, and A. Evans, A guide to government in Afghanistan. University of Arizona Libraries, 2004.

[5] A. R. Zia, "The World Bank Group in Afghanistan," World Bank, no. 052, p. 27, 2018.

[6] J. W. Michael, Facebook Graph API Development with Flash, 1st ed. Birmingham, B27 6PA, UK: Packt Publishing Ltd, 2010.

[7] S. Gabriel, Beginning Google Maps API 3. US: Springer Science+Business Media, LLC., 233 Spring Street, 6th Floor, New York, 2010. 
[8] "Messenger Platform." [Online]. Available: https://developers.facebook.com/docs/messengerplatform. [Accessed: 02-Aug-2019].

[9] "Developer Guide | Geolocation API," Google Developers. [Online]. Available: https://developers.google.com/maps/documentation/geol ocation/intro. [Accessed: 29-Jul-2019].

[10] "Developer Guide | Geolocation API," Google Developers. [Online]. Available: https://developers.google.com/maps/documentation/geol ocation/intro. [Accessed: 02-Aug-2019].

[11] tutorialspoint.com, "Google Maps Tutorial Point." Tutorials Point Pvt. Ltd., 2014.

[12] "How PubNub Works," PubNub. [Online]. Available: https://www.pubnub.com/developers/tech/how-pubnubworks/. [Accessed: 02-Aug-2019].

[13] "Graph API - Documentation," Facebook for Developers. [Online]. Available: https://developers.facebook.com/docs/graph-api/. [Accessed: 02-Aug-2019]. 